\title{
Confocal Scanning Raman Spectroscopy (CSRS) Of An Operating Organic Light-Emitting Diode
}

\author{
Beynor Antonio Paez-Sierra ${ }^{\mathrm{a}, \mathrm{b}}$ and Henrique Leonel Gomes ${ }^{\mathrm{c}}$ \\ ${ }^{a}$ QUBITON Laboratories, e-mail: beynor.paez@qubiton.com, Julius Raab 1-3 A4040 Linz, Austria. \\ ${ }^{b}$ NANOIDENT Technologies AG,Untere Danaulaende 21-25 A4020 Linz, Austria. \\ ${ }^{c}$ Universidade do Algarve, Center of Electronic Optoelectronics and Telecommunications (CEOT), \\ Campus de Gambelas, 8000 Faro, Portugal
}

\section{INTRODUCTION AND EXPERIMENTAL CONDITIONS}

Organic molecules with semiconducting properties are becoming nowadays core of the organic-based electronic era. Although organic light emitting diodes (OLEDs) have already matured for commercial applications, they still require longer device lifetimes. Some of the long-standing challenges in OLED technology relay on degradation and failure mechanisms. Several authors observed that degradation and subsequent damage of OLEDs is accompanied by formation of dark non-emissive spots [1-2]. Implementation of the confocal scanning Raman spectroscopy (CSRS) measurements helps to understand the chemistry, physics of OLEDs and moreover to have better confidence on their quality assurance. CRCS advantages other local probes where the information might be limited to the spotted region or to large excitation energies. The non-invasive vibrational analysis permits energetically to resolve finestructure features, i.e. a $1000 \mathrm{~cm}^{-1}$ Raman shift corresponds to about $123.9 \mathrm{meV}$. Here the analysis resolves energies as better as $10 \mathrm{meV}$. The device architecture reported here and CSRS scheme are illustrated in FIGURE 1.

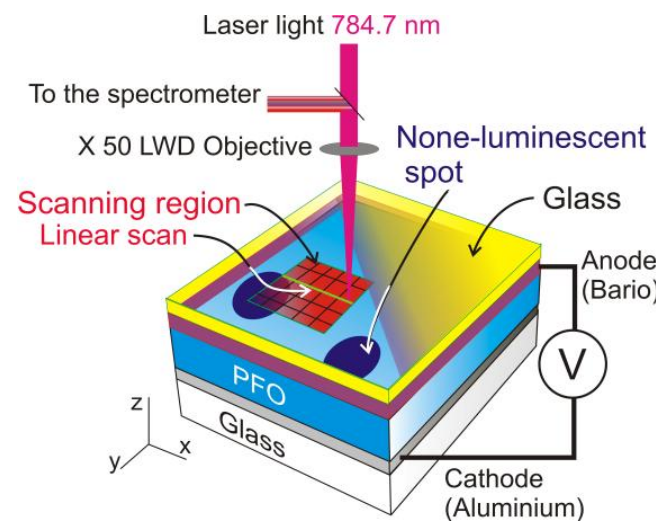

(a)

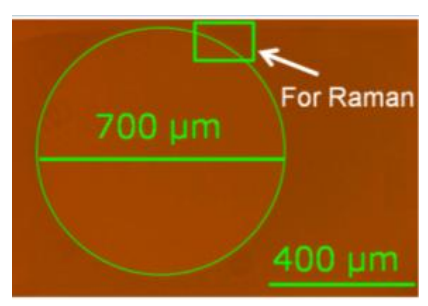

(b)

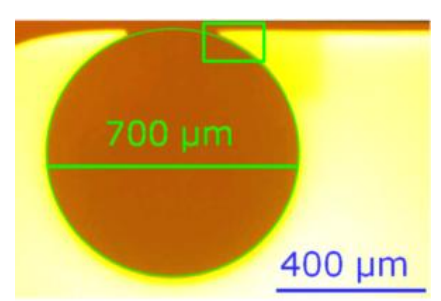

(c)

FIGURE 1. Experimental conditions. (a) Device architecture and configuration of the confocal scanning Raman spectrometer. (b) False color-coded picture of the PFO layer and (b) optical image of the OLED under operation where the bright region corresponds to the emitting layer. 
The CSRS maps were carried out without an under OLED operation covering two typical regions, named emissive and non-emissive spots.

\section{CSRS Maps of the OLED under Operation}

The ex situ analysis of the OLEDs were performed in normal room conditions. FIGURE 2 illustrates a typical linear CSRS map performed from the emissive points towards non-emissive ones. The non-emissive spots presented higher Raman intensity in comparison with the luminescent ones. The phenomenon is associated with an increase in the PFO $\pi-\pi^{*}$ absorption band which becomes favorable to the excitation wavelength, thus the Raman spectrum is enhanced.

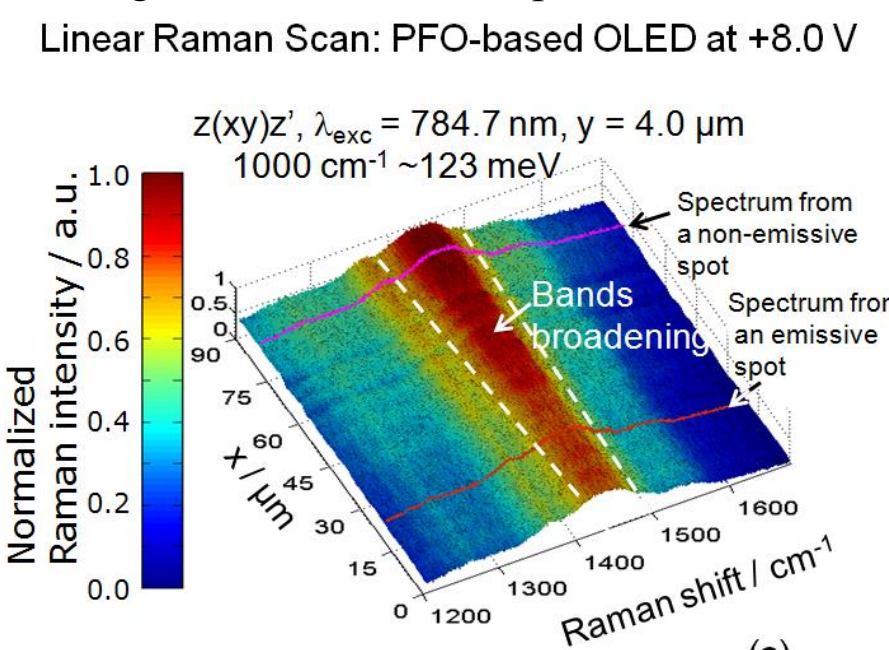

(a)

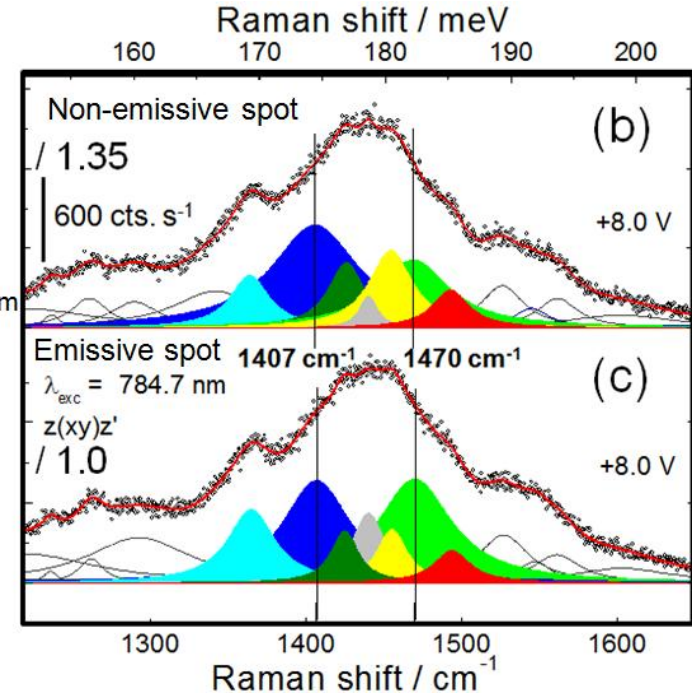
FIGURE 2. CSRS of the OLED operating at $+8.0 \mathrm{~V}$. (a) Color-coded linear CSRS map performed at
the organic layer. (b)-(c) Representative normalized Raman spectra of the non-emissive and emissive spots with the corresponding deconvoluted bands, respectively.

In addition, the CSRS maps revealed progressive band broadening from emissive spots towards non-emissive zones, which are well-pronounced in the spectral region $[1300-1600] \mathrm{cm}^{-1}$. The integrated Raman intensity ratio of the deconvoluted band at $1407.0 \mathrm{~cm}^{-1}$ to the band at $1470.0 \mathrm{~cm}^{-1}$ was found to be proportional to the number of molecules that might emit and hence an indicator of integrity of the electroluminescent layer.

\section{ACKNOWLEDGMENTS}

We would like to thank Dago de Leeuw for providing the samples and to Fundação para a Ciência e Technology, research unit 631, CEOT.

\section{REFERENCES}

1. J. S. Kim, P. K. H. Ho, C. E. Murphy, N. Baynes and R. H. Friend, Adv. Mater. 14, 206-209 (2002).

2. E. Giorgetti, G. Margheri, T. Delrosso, S. Sottini, M. Muniz-Miranda, M. Innocenti, Appl. Phys. B 79, 603-609 (2004). 